\title{
Ramsey Graphs for a Star on Three Vertices Versus a Cycle
}

\author{
Maya Nabila*, Edy Tri Baskoro, Hilda Assiyatun
}

\author{
Combinatorial Mathematics Research Group, Faculty of Mathematics and Natural Sciences, \\ Institut Teknologi Bandung, Indonesia \\ Corresponding author. Email: mayanabila@students.itb.ac.id
}

\begin{abstract}
Let $G, A$, and $B$ be simple graphs. The notation $G \rightarrow(A, B)$ means that for any red-blue coloring of the edges of $G$, there is a red copy of $A$ or a blue copy of $B$ in $G$. A graph $G$ is called a Ramsey graph for $(A, B)$ if $G \rightarrow(A, B)$. Additionally, if the graph $G$ satisfies that $G-e \nrightarrow(A, B)$, for any $e \in E(G)$, then $G$ is called a Ramsey $(A, B)$-minimal graph. The set of all Ramsey $(A, B)$-minimal graphs is denoted by $\mathcal{R}(A, B)$. In this paper, we study the Ramsey $\left(C_{n}, K_{1,2}\right)$-minimal graphs. Specifically, we give some Ramsey $\left(C_{n}, K_{1,2}\right)$-minimal graphs for any $n \in[7,10]$. We also construct Ramsey $\left(C_{n}, K_{1,2}\right)$-graphs from the well-known Harary graph, for any integer $n \geq 6$.
\end{abstract}

Keywords: Ramsey minimal graph, Star, Cycle.

\section{INTRODUCTION}

Frank Plumpton Ramsey introduced a Ramsey theory which enables us to determine whether a given set of logical statements is consistent or not. Naturally, the study of Ramsey theory has been further developed. One of them is the study of constructing Ramsey minimal graphs, introduced by Burr in 1970 [1].

The notation $G \rightarrow(A, B)$ means that for any red-blue coloring of the edges of $P$ there is a red copy of $A$ or a blue copy of $B$ in $G$. A graph $G$ is called a Ramsey graph for $(A, B)$ if $G \rightarrow(A, B)$. Additionally, if $G-e \nrightarrow(A, B)$ holds for any $e \in E(G)$, then $G$ is called a Ramsey (A, B)-minimal graph. The set of all Ramsey $(G, H)$ minimal graphs is denoted by $\mathcal{R}(G, H)$. A pair of $(G, H)$ is called Ramsey-finite if the set $\mathcal{R}(G, H)$ is finite, otherwise the pair $(G, H)$ is called Ramsey-infinite. The closure of graph $G$ is the graph obtained from $G$ by recursively joining pairs of nonadjacent vertices whose the degree sum is at least $|G|$ until no such pair remains. We include some classic theorems about hamiltonicity that will be used in this paper.

Theorem 1.1 [2] If $G$ is a simple graph with $|G| \geq 3$ and $\delta(G) \geq \frac{|G|}{2}$, then $G$ is hamiltonian.

Theorem 1.2 [3] A simple graph is hamiltonian if and only if its closure is hamiltonian.
There are some previous results about Ramsey minimal graphs, for instance in [1] Burr et al. proved that if $G$ and $H$ are both disjoint unions of edges then the pair $(G, H)$ is Ramsey-finite. Furthermore, in [4], they proved that the pair $\left(m K_{2}, H\right)$ is Ramsey-finite for $m \geq 1$ and any graph $H$. Nesetril and Rodl [5] showed that the pair $(G, H)$ is Ramsey infinite if both $G$ and $H$ are 3connected or if $G$ and $H$ are forests neither of which is a union of stars. In 2010, Vetrik et al. [6] gave an infinite family of Ramsey $\left(K_{1,2}, C_{4}\right)$-minimal graphs of diameter greater than or equals 4 .

In [7], they constructed new infinite class of Ramsey $\left(K_{1,2}, C_{4}\right)$-minimal graphs. In the same year, Nabila and Baskoro [8] constructed Ramsey $\left(C_{n}, K_{1,2}\right)$ - minimal graphs for $n=5,6,8$. They also gave Ramsey $\left(C_{n}, K_{1,2}\right)$ graphs for even $n \in[10,18]$. In this paper, we give Ramsey $\left(C_{n}, K_{1,2}\right)$-minimal graphs for some $n \in[7,10]$, and derive Ramsey $\left(C_{n}, K_{1,2}\right)$ graphs from the wellknown Harary graphs, for any integer $n \geq 6$.

\section{MAIN RESULTS}

\subsection{Ramsey $\left(C_{n}, K_{1,2}\right)$-minimal}

In this section, we give a new graph in the set $\mathcal{R}\left(C_{n}, K_{1,2}\right)$, for $n=7,8,9$, and 10. Define the graphs $F_{1}, F_{2}, F_{3}$, and $F_{4}$ as depicted in Figure 1. 


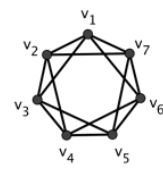

i) $F_{1}$

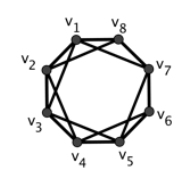

ii) $F_{2}$

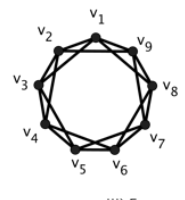

iii) $F_{3}$

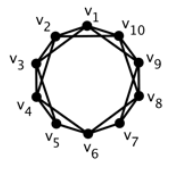

iv) $F_{4}$
Figure 1 Graphs $F_{1}, F_{2}, F_{3}, F_{4}$.

Theorem 2.1 $F_{1} \in \mathcal{R}\left(C_{7}, K_{1,2}\right), F_{2} \in \mathcal{R}\left(C_{8}, K_{1,2}\right), F_{3} \in$ $\mathcal{R}\left(C_{9}, K_{1,2}\right)$, and $F_{4} \in \mathcal{R}\left(C_{10}, K_{1,2}\right)$.

Proof.

i. We will show that $F_{1} \rightarrow\left(C_{7}, K_{1,2}\right)$. Consider any red-blue coloring on graph $F_{1}$ that does not contain a blue copy of $K_{1,2}$. Since there is no blue copy of $K_{1,2}$, then all blue edges will form a matching $M$ as a subgraph of some maximal blue matching shown in Figure 2. Then, we obtain a red copy of $C_{7}=$ $(1,2,3,4,5,6,7)$ in $F_{1}$ for any matching $M$.
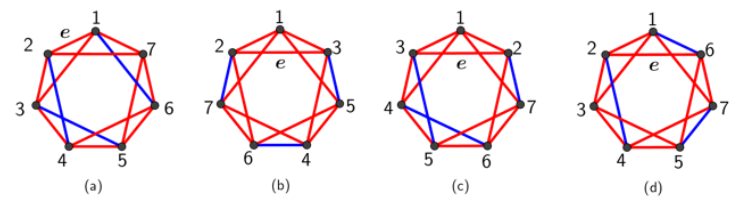

Figure 2 Graph $F_{1} \rightarrow\left(C_{7}, K_{1,2}\right)$.

Next, we will show the minimality of $F_{1}$. For any edge $e \in E\left(F_{1}\right)$, consider the graph $F_{1}-e$. By isomorphism, we can assume that $e=v_{1} v_{2}$ or $e=$ $v_{2} v_{7}$. If $e=v_{1} v_{2}$ then use the coloring in Figure 2 (a) by removing the edge $e$. It is clear that this redblue coloring contain neither blue copy of $K_{1,2}$ nor red copy of $C_{7}$. Therefore, we obtain that $F_{1}-e \rightarrow$ $\left(C_{7}, K_{1,2}\right)$ for $e=v_{1} v_{2}$. Similarly, if $e=v_{2} v_{7}$, use the coloring in Figure 2(b). Thus, $F_{1} \in \mathcal{R}\left(C_{7}, K_{1,2}\right)$.

ii. We will show that $F_{2} \rightarrow\left(C_{8}, K_{1,2}\right)$. Consider any red-blue coloring on graph $F_{2}$ that does not contain a blue copy of $K_{1,2}$. Since there is no blue copy of $K_{1,2}$, then all blue edges will form a matching $M$ as a subgraph of some maximal blue matching shown in Figure 3. Then, we obtain a red copy of $C_{8}=$ $(1,2,3,4,5,6,7,8)$ in $F_{2}$ for any matching $M$.

Next, we will show the minimality of $F_{2}$. For any edge $e \in E\left(F_{2}\right)$, consider the graph $F_{2}-e$. By isomorphism, we can assume that $e=v_{2} v_{8}, e=$ $v_{3} v_{4}$ or $e=v_{7} v_{8}$. If $e=v_{2} v_{8}$ then use the coloring in Figure 3(a) by removing the edge $e$. It is clear that this red-blue coloring contain neither blue copy of $K_{1,2}$ nor red copy of $C_{8}$. Therefore, we obtain that $F_{2}-e \nrightarrow\left(C_{8}, K_{1,2}\right)$ for $e=v_{2} v_{8}$. Similarly, if $e=$ $v_{3} v_{4}$, use the coloring in Figure 3(b), and if $e=$ $v_{7} v_{8}$, use the coloring in Figure 3(e). Thus, $F_{2} \in$ $\mathcal{R}\left(C_{8}, K_{1,2}\right)$.

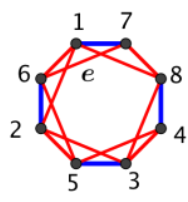

(a)

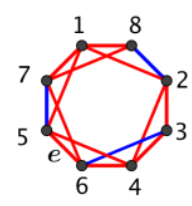

(b)

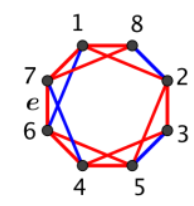

(c)

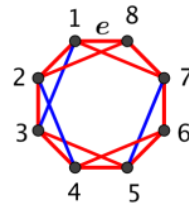

(d)

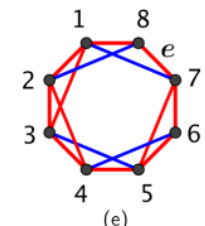

Figure 3 Graph $F_{2} \rightarrow\left(C_{8}, K_{1,2}\right)$.

Next, we will show the minimality of $F_{2}$. For any edge $e \in E\left(F_{2}\right)$, consider the graph $F_{2}-e$. By isomorphism, we can assume that $e=v_{2} v_{8}, e=$ $v_{3} v_{4}$ or $e=v_{7} v_{8}$. If $e=v_{2} v_{8}$ then use the coloring in Figure 3(a) by removing the edge $e$. It is clear that this red-blue coloring contain neither blue copy of $K_{1,2}$ nor red copy of $C_{8}$. Therefore, we obtain that $F_{2}-e \nrightarrow\left(C_{8}, K_{1,2}\right)$ for $e=v_{2} v_{8}$. Similarly, if $e=$ $v_{3} v_{4}$, use the coloring in Figure 3(b), and if $e=$ $v_{7} v_{8}$, use the coloring in Figure 3(e). Thus, $F_{2} \in$ $\mathcal{R}\left(C_{8}, K_{1,2}\right)$.

iii. We will show that $F_{3} \rightarrow\left(C_{9}, K_{1,2}\right)$. Consider any red-blue coloring on graph $F_{3}$ that does not contain a blue copy of $K_{1,2}$. Since there is no blue copy of $K_{1,2}$, then all blue edges will form a matching $M$ as a subgraph of some maximal blue matching shown in Figure 4. Then, we obtain a red copy of $C_{9}=$ $(1,2,3,4,5,6,7,8,9)$ in $F_{3}$ for any matching $M$.
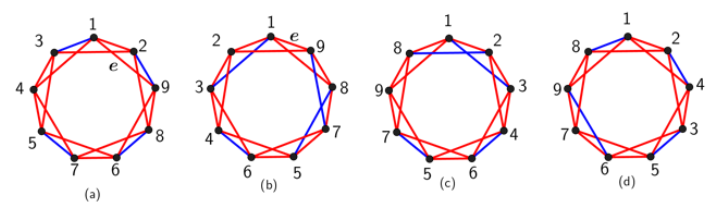

Figure 4 Graph $F_{3} \rightarrow\left(C_{9}, K_{1,2}\right)$.

Next, we will show the minimality of $F_{3}$. For any edge $e \in E\left(F_{3}\right)$, consider the graph $F_{3}-e$. By isomorphism, we can assume that $e=v_{1} v_{8}$ or $e=$ $v_{1} v_{2}$. If $e=v_{1} v_{8}$ then use the coloring in Figure 4(a) by removing the edge $e$. It is clear that this redblue coloring contain neither blue copy of $K_{1,2}$ nor red copy of $C_{9}$. Therefore, we obtain that $F_{3}-e \rightarrow$ $\left(C_{9}, K_{1,2}\right)$ for $e=v_{1} v_{3}$. Similarly, if $e=v_{1} v_{2}$, use the coloring in Figure 4(b). Thus, $F_{3} \in \mathcal{R}\left(C_{9}, K_{1,2}\right)$.

iv. We will show that $F_{4} \rightarrow\left(C_{10}, K_{1,2}\right)$. Consider any red-blue coloring on graph $F_{4}$ that does not contain a blue copy of $K_{1,2}$. Since there is no blue copy of $K_{1,2}$, then all blue edges will form a matching $M$ as a subgraph of some maximal blue matching shown 
in Figure 5. Then, we obtain a red copy of $C_{10}=$ $(1,2,3,4,5,6,7,8,9,10)$ in $F_{1}$ for any matching $M$.<smiles></smiles>

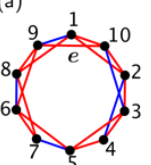

(e)

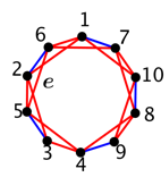

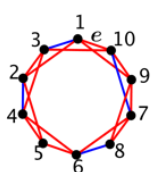

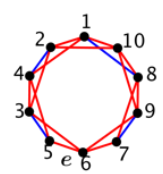

(d)

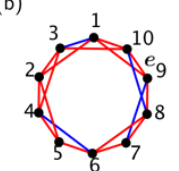

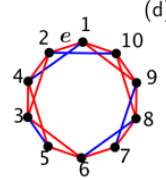

(g)
Figure 5 Graph $F_{4} \rightarrow\left(C_{10}, K_{1,2}\right)$.

Next, we will show the minimality of $F_{4}$. For any edge $e \in E\left(F_{4}\right)$, consider the graph $F_{4}-e$. By isomorphism, we can assume that $e=v_{1} v_{3}, e=$ $v_{2} v_{4}, e=v_{1} v_{10}$ or $e=v_{5} v_{6}$. If $e=v_{1} v_{3}$ then use the coloring in Figure 5(a) by removing the edge $e$. It is clear that this red-blue coloring contain neither blue copy of $K_{1,2}$ nor red copy of $C_{8}$. Therefore, we obtain that $F_{4}-e \rightarrow\left(C_{10}, K_{1,2}\right)$ for $e=v_{1} v_{3}$. Similarly, if $e=v_{2} v_{4}$, use the coloring in Figure 5 (b), if $e=v_{1} v_{10}$, use the coloring in Figure 5(c), and if $e=v_{5} v_{6}$, use the coloring in Figure 5(d). Thus, $F_{4} \in \mathcal{R}\left(C_{10}, K_{1,2}\right)$.

\subsection{The Ramsey $\left(C_{n}, K_{1,2}\right)$ graph for $n \geq 6$}

In this section, we construct new Ramsey graphs (not necessarily minimal) for a pair of $\left(C_{2 n}, K_{1,2}\right)$ from the well-known Harary graph $H_{n, m}$, namely an $n$-connected with $m$ vertices that has exactly $\{n m / 2\}$ edges. The structure $H_{n, m}$ depends on the parities of $n$ and $m$. For this construction, we use only the Harary graph $H_{n, m}$ with even $m$.

If both $m$ and $n$ are even and $n=2 k$ for some $k$, then the definition of the Harary graph $H_{2 k, m}$ is as follows.

Definition 1 Graph $H_{2 k, m}$ has $v_{1}, v_{2}, \cdots, v_{m-1}$ vertices. Two vertices $v_{i}$ and $v_{j}$ are adjacent if $|j-i| \leq k$ (where addition is taken modulo $m$ ).

If $m$ is even and $n$ is odd and $n=2 k+1$ for some $k$, then the definition of the Harary graph $H_{2 k+1, m}$ is as follows.

Definition 2 Graph $H_{2 k+1, m}$ is constructed by first drawing $H_{2 k, m}$ and then adding edges joining vertex $v_{i}$ to vertex $v_{i+m / 2}$ for $1 \leq i \leq n / 2$.

Now, first we construct Ramsey graphs for a pair of $\left(C_{2 n}, K_{1,2}\right)$ by modifying the Harary graph $H_{n, 2 n}$ if $n$ is odd. Our modified graph $H_{n, 2 n}^{*}$ is defined as follows.

Definition 3 Let $n \geq 7$ be an odd integer. The graph $H_{n, 2 n}^{*}$ is a graph that we obtain by modifying graph $H_{n, 2 n}$ as below.

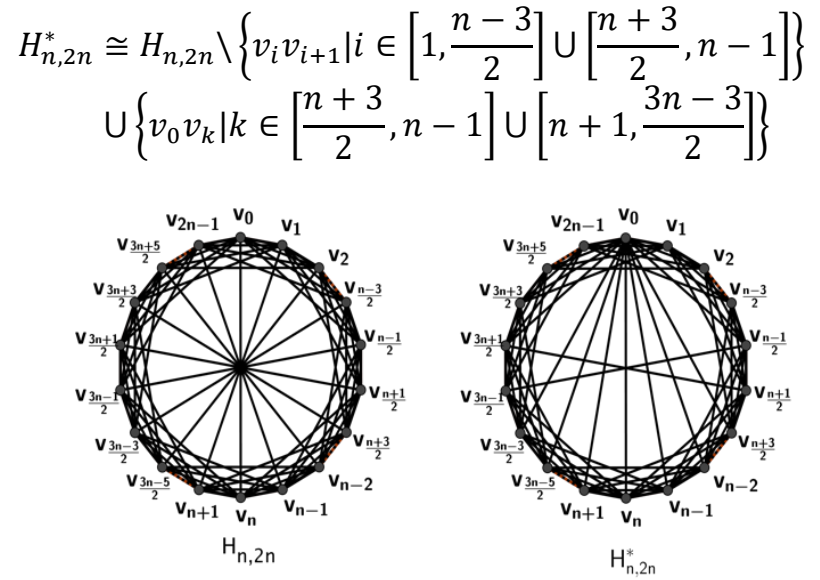

Theorem 2.2 $H_{n, 2 n}^{*} \rightarrow\left(C_{2 n}, K_{1,2}\right)$ for odd $n \geq 7$.

Proof. We will show that $H_{n, 2 n}^{*} \rightarrow\left(C_{2 n}, K_{1,2}\right)$. Consider any red-blue coloring of the edges of graph $H_{n, 2 n}^{*}$ containing no blue copy of $K_{1,2}$. Then, all the blue edges will form a matching $M$. Now, consider the graph graph $H_{n, 2 n}^{*} \backslash M$.

(i) Partition the vertices of the graph $H_{n, 2 n}^{*} \backslash M$ into 8 sets as follows.

$$
\begin{aligned}
& A_{0}=\left\{v_{0}\right\} ; \\
& A_{1}=\left\{v_{1}, v_{2}, \ldots, \frac{v_{n-3}}{2}\right\} ; \\
& A_{2}=\left\{v_{\frac{n-1}{2}}, \frac{v_{n+1}}{2}\right\} ; \\
& A_{3}=\left\{v_{\frac{n+3}{2}}, \ldots, v_{n-1}\right\} ; \\
& A_{4}=\left\{v_{n}\right\} ; \\
& A_{5}=\left\{v_{n+1}, \ldots, \frac{v_{3 n-3}}{2}\right\} ; \\
& A_{6}=\left\{v_{\frac{3 n-1}{2}}, \frac{v_{3 n+1}}{2}\right\} ; \\
& A_{7}=\left\{v_{\frac{3 n+3}{2}}, \ldots, v_{2 n-1}\right\} ; \\
& \text { where }\left|A_{i}\right|=\frac{n-3}{2} \text { for } i \in\{1,3,5,7\} . \\
& \text { Note } \quad \text { that } \quad d\left(v_{0}\right)=2 n-4, \quad d\left(v_{n-1}\right)= \\
& d\left(v_{n+1}^{2}\right)=d\left(v_{\frac{3 n-1}{2}}^{2}\right)=d\left(\frac{v_{3 n-1}}{2}\right)=n-1, \\
& \forall v \in A_{1}, A_{7}, d(v)=n-2, \text { and } \forall v \in A_{3}, A_{5}, \\
& d(v)=n-1 .
\end{aligned}
$$

Therefore, we obtain the degree sequence of graph $H_{n, 2 n}^{*} \backslash M$ as follows.

$$
2 n-4, \underbrace{n-1, \ldots, n-1}_{n+2}, \underbrace{n-2, \ldots, n-2}_{n-3} .
$$

(ii) Next, do the closure process of the graph $H_{n, 2 n}^{*} \backslash M$. In the first step of the closure, the vertex 
$v_{0}$ will be adjacent to $v=v_{\frac{n+1}{2}}$ and $v=\frac{v_{3 n-1}}{2}$, since their degree sum of both vertices is $d(v)+$ $d\left(v_{0}\right)=n-1+2 n-4 \geq 2 n$. Therefore, their degrees will increase as follows:

$$
\begin{aligned}
& d\left(v_{0}\right)=2 n-2 ; \\
& d\left(\frac{v_{n+1}}{2}\right)=n ; \\
& d\left(\frac{v_{\frac{3 n-1}{2}}}{2}\right)=n .
\end{aligned}
$$

Consequently, after the first step, we have the degree sequence:

$$
2 n-2, n, n, \underbrace{n-1, \ldots, n-1}_{n}, \underbrace{n-2, \ldots, n-2}_{n-3} .
$$

(iii) Since vertex $\frac{v_{n+1}}{2}$ and vertex $\frac{v_{3 n-1}}{2}$ are not adjacent and $d\left(\frac{v_{n+1}}{2}\right)+d\left(v_{\frac{3 n-1}{2}}\right)^{2}=n+n \geq$ $2 n$, then these vertices will be adjacent in the second step of our closure process. This makes their degrees change into:

$d\left(\frac{v_{n+1}}{2}\right)=n+1$ and $d\left(\frac{v_{3 n-1}}{2}\right)=n$.

Thus, the degree sequence becomes:

$2 n-2, n+1, n+1, \underbrace{n-1, \ldots, n-1}_{n}, \underbrace{n-2, \ldots, n-2}_{n-3}$.

(iv) Now, consider vertex $\frac{v_{n+1}}{2}$ and vertex $v$ on $A_{5}$, we can see that $d\left(\frac{v_{n+1}}{2}\right)+d(v)=(n+1)+$ $(n-1) \geq 2 n$. This means that in the next step of the closure process on, we can consider these vertices: $\frac{v_{n+1}}{2}$ and $\mathrm{v}$, for all $v \in A_{5}$. So, their degrees will change into:

$d\left(\frac{v_{n+1}}{2}\right)=\frac{3 n-1}{2}$

$d\left(\frac{v_{3 n-1}}{2}\right)=n, \forall v \in A_{5}$.

Thus, the degree sequence becomes:

$$
\begin{aligned}
& 2 n-2, \frac{3 n-1}{2}, n+1, \underbrace{n, \ldots, n}_{\frac{n-3}{2}}, \underbrace{n-1, \ldots, n-1}_{\frac{n+3}{2}}, \\
& \underbrace{n-2, \ldots, n-2}_{n-3} .
\end{aligned}
$$

(v) In the next step, consider vertex $\frac{v_{n+1}}{2}$ and vertex $v$ on $A_{7}$. We can see that

$$
d\left(\frac{v_{n+1}}{2}\right)+d(v)=\frac{3 n-1}{2}+(n-1) \geq 2 n .
$$

By the closure process, their degrees will become:

$d\left(\frac{v_{n+1}}{2}\right)=2 n-2$; $d\left(v_{\frac{3 n-1}{2}}\right)=n-1, \forall v \in A_{7}$.

The degree sequence will become:

$$
\begin{aligned}
& 2 n-2,2 n-2, n+1, \underbrace{n, \ldots, n}_{\frac{n-3}{2}}, \underbrace{n-1, \ldots, n-1}_{n}, \\
& \underbrace{n-2, \ldots, n-2}_{\frac{n-3}{2}} .
\end{aligned}
$$

(vi) Now, consider vertex $\frac{v_{3 n-1}}{2}$ and vertex $v$ on $A_{3}$. The degree sum will be:

$$
d\left(\frac{v_{3 n-1}}{2}\right)+d(v)=(n+1)+(n-1) \geq 2 n .
$$

Therefore, these vertices will be adjacent in our closure process. So, the degrees of these vertices become:

$d\left(\frac{v_{3 n-1}}{2}\right)=\frac{3 n-1}{2}$;

$d(v)=n, \quad \forall v \in A_{3}$.

As the consequence, the degree sequence becomes:

$$
\begin{aligned}
& 2 n-2,2 n-2, \frac{3 n-1}{2}, \underbrace{n, \ldots, n}_{n-3}, \underbrace{n-1, \ldots, n-1}_{\frac{n+3}{2}}, \\
& \underbrace{n-2, \ldots, n-2}_{\frac{n-3}{2}} .
\end{aligned}
$$

(vii) Consider vertex $\frac{v_{3 n-1}}{2}$ and vertex $v$ on $A_{1}$, we can see that

$$
d\left(\frac{v_{3 n-1}}{2}\right)+d(v)=\left(\frac{3 n-1}{2}\right)+(n-2) \geq 2 n .
$$

Then, after the closure process their degrees become:

$d\left(v_{\frac{3 n-1}{2}}\right)=2 n-2$;

$d(v)=n-1, \quad \forall v \in A_{1}$.

As the consequence, the degree sequence also change into

$$
\underbrace{2 n-2,, \ldots, 2 n-2}_{3}, \underbrace{n, \ldots, n}_{n-3}, \underbrace{n-1, \ldots, n-1}_{n} .
$$

(viii) Now, consider vertex $\frac{v_{n+3}}{2}$ and vertex $v$ on $A_{5} \backslash$ $\left\{v_{n+1}\right\}$, we can see that $d\left(v_{\frac{n+3}{2}}\right)+d(v)=n+$ $n \geq 2 n$. From the closure process on these vertices, we obtain:

$d\left(\frac{v_{n+3}}{2}\right)=\frac{3 n-5}{2}$

$d(v)=n+1, \forall v \in A_{5} \backslash\left\{v_{n+1}\right\}$

This implies that the degree sequence becomes 


$$
\begin{aligned}
& \underbrace{2 n-2,, \ldots, 2 n-2}_{3}, \frac{3 n-5}{2}, \underbrace{n+1, \ldots, n+1}_{\frac{n-3}{2}-1}, \\
& \underbrace{n, \ldots, n}_{\frac{n-3}{2}}, \underbrace{n-1, \ldots, n-1}_{n},
\end{aligned}
$$

(ix) Now, consider vertex $\frac{v_{3 n-3}}{2}$ and vertex $v$ on $A_{1} U$ $\left\{v_{\frac{n-1}{2}}\right\}$. We can see that

$$
d\left(\frac{v_{3 n-3}}{2}\right)+d(v)=(n+1)+(n-1) \geq 2 n .
$$

From the closure process on these vertices, we obtain:

$d\left(\frac{v_{3 n-3}}{2}\right)=\frac{3 n+1}{2}$

$d(v)=n, \forall v \in A_{1} \cup\left\{v_{\frac{n-1}{2}}\right\}$.

Then, the degree sequence becomes:

$$
\begin{aligned}
& \underbrace{2 n-2,, \ldots, 2 n-2}_{3}, \frac{3 n+1}{2}, \frac{3 n-5}{2}, \\
& \underbrace{n+1, \ldots, n+1}_{\frac{n-3}{2}-2}, \underbrace{n, \ldots, n}_{n-2}, \underbrace{n-1, \ldots, n-1}_{\frac{n+1}{2}}
\end{aligned}
$$

(x) Consider vertex $\frac{v_{n+3}}{2}$ and vertex $v$ on $A_{7} U$ $\left\{\frac{v_{3 n+1}}{2}\right.$, we can see that

$$
d\left(v_{n+3}\right)+d(v)=\frac{3 n-5}{2}+(n-1) \geq 2 n .
$$

The closure process on these vertices will result:

$d\left(\frac{v_{n+3}}{2}\right)=2 n-3 ;$

$d(v)=n, \forall v \in A_{7} \cup\left\{\frac{v_{3 n+1}}{2}\right\}$.

Then, the degree sequence becomes:

$$
\begin{aligned}
& \underbrace{2 n-2,, \ldots, 2 n-2}_{3}, 2 n-3, \frac{3 n+1}{2}, \\
& \underbrace{n+1, \ldots, n+1}_{\frac{n-3}{2}-2}, \underbrace{n, \ldots, n}_{\frac{n n-5}{2}}, n-1 .
\end{aligned}
$$

(xi) Consider vertex $\frac{v_{n-1}}{2}$ and vertex $\frac{v_{3 n+1}}{2}$. We observe that $d\left(\frac{v_{n-1}}{2}\right)+d\left(\frac{v_{3 n+1}}{2}\right)=n+n \geq$ $2 n$.

By the closure process on these vertices, we have:

$d\left(\frac{v_{n-1}}{2}\right)=n+1$;

$d\left(\frac{v_{3 n+1}}{2}\right)=n+1$.

Then, the degree sequence becomes:

$$
\begin{gathered}
\underbrace{2 n-2, \ldots, 2 n-2}_{3}, 2 n-3, \frac{3 n+1}{2}, \\
\underbrace{n+1, \ldots, n+1}_{\frac{n-3}{2}}, \underbrace{n, \ldots, n-1 .}_{\frac{n, \ldots, n}{2}-2}, n-1 .
\end{gathered}
$$

(xii) Lastly, consider vertex $\frac{v_{n-1}}{2}$ and vertex $v_{n}$. We can see that

$$
d\left(v_{\frac{n-1}{2}}\right)+d\left(v_{n}\right)=(n+1)+(n-1) \geq 2 n .
$$

The closure process will result

$$
\begin{aligned}
& d\left(\frac{v_{n-1}}{2}\right)=n+2 ; \\
& d\left(v_{n}\right)=n .
\end{aligned}
$$

Then, the degree sequence becomes:

$$
\begin{gathered}
\underbrace{2 n-2,, \ldots, 2 n-2}_{3}, 2 n-3, \frac{3 n+1}{2}, n+2 \\
\underbrace{n+1, \ldots, n+1}_{\frac{n-5}{2}}, \underbrace{n, n, n}_{\frac{3 n-5}{2}-1} .
\end{gathered}
$$

Since the degree of each vertex is at least $n$ in the closure of $H_{n, 2 n}^{*} \backslash M$, then by Theorem 1.1, we obtain $c\left(H_{n, 2 n}^{*} \backslash M\right)$ is hamiltonian. By Theorem 1.2, we obtain $H_{n, 2 n}^{*} \backslash M$ is hamiltonian. So that, $H_{n, 2 n}^{*} \backslash M$ contains a red copy of $C_{2 n}$. Therefore, $H_{n, 2 n}^{*} \rightarrow\left(C_{2 n}, K_{1,2}\right)$ for odd $n \geq 7$.

Now, we construct Ramsey graphs for a pair of $\left(C_{2 n}, K_{1,2}\right)$ by modifying the Harary graph $H_{n, 2 n}$ if $n$ is even. Our modified graph $H_{n, 2 n}^{* *}$ is defined as follows.

Definition 4 Let $n \geq 6$ be an even integer. The graph $H_{n, 2 n}^{* *}$ is a graph that we obtain by modifying graph $H_{n, 2 n}$ as below.

$$
\begin{aligned}
H_{n, 2 n}^{* *} & \cong H_{n, 2 n} \backslash\left\{v_{i} v_{i+\frac{n}{2}} \mid i \in\left[0, \frac{n}{2}-1\right] \cup\left[n, \frac{3 n}{2}-1\right]\right\} \\
& \cup\left\{v_{j} v_{j+n} \mid j \in\left[\frac{n}{2}-1, \frac{n}{2}\right]\right\} \\
& \cup\left\{v_{0} v_{k} \mid k \in\left[\frac{n}{2}+1, \frac{3 n}{2}-2\right]\right\}
\end{aligned}
$$
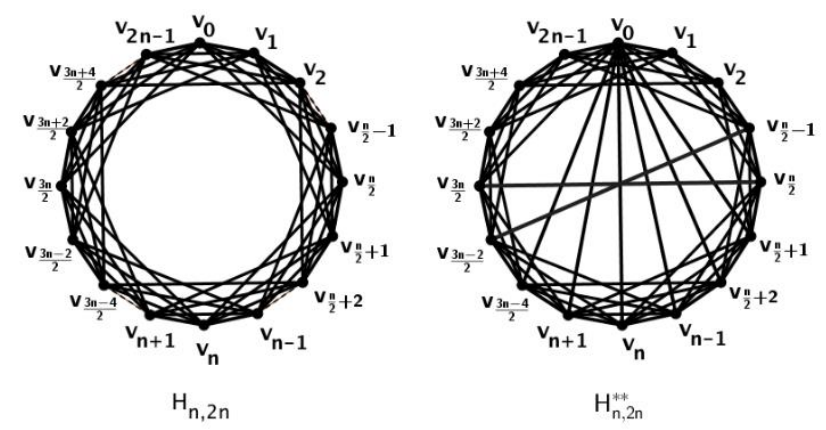

Theorem $2.3 H_{n, 2 n}^{* *} \rightarrow\left(C_{2 n}, K_{1,2}\right)$ for even $n \geq 6$.

Proof. We will show that $H_{n, 2 n}^{* *} \rightarrow\left(C_{2 n}, K_{1,2}\right)$. Consider any red-blue coloring on the edges of the graph $H_{n, 2 n}^{* *}$ 
containing no blue copy of $K_{1,2}$. Then, all the blue edges will form a matching $M$. It can be verified that every vertex in the closure of the graph $H_{n, 2 n}^{* *} \backslash M$ has degree at least $n$. Then $H_{n, 2 n}^{* *} \backslash M$ is hamiltonian (by Theorem 1.1 and 1.2) Therefore, there exists a blue copy of $C_{2 n}$ in $H_{n, 2 n}^{* *} \backslash M$. Therefore, $H_{n, 2 n}^{* *} \rightarrow\left(C_{2 n}, K_{1,2}\right)$.

\section{AUTHORS' CONTRIBUTIONS}

Conseptualization, M.N., E.T.B., and H.A.; validation, M.N., E.T.B., and H.A.; writing original - draft preparation, M.N.; writing - review, E.T.B. and H.A.; funding acquisition, E.T.B. All authors have read and agreed to the published version of the manuscript.

\section{ACKNOWLEDGMENTS}

This research has been supported by the Indonesian Ministry of Education, Culture, Research and Technology under the program of "Pendidikan Magister menuju Doktor untuk Sarjana Unggulan (PMDSU)".

\section{REFERENCES}

[1] S.A. Burr, P. Erdos, L. Lovasz, On graphs of Ramsey type, vol. 1, Ars Combinatoria, 1976, pp. 167-190.
[2] G.A. Dirac, Some Theorem on Abstract Graphs, vol. 3, Proceedings of the London Mathematical Society, 1952, pp. 69-81.

[3] J.A. Bondy, U.S.R. Murty, Graph Theory with Applications, vol. 290, Macmillan London, 1976.

[4] S.A. Burr, P. Erdos, R.J. Faudree, C.C. Rousseau, R.H. Schelp, A class of Ramsey-finite graphs, Proc. $9^{\text {th }}$ SE Conf. on Combinatorics, Graph theory and Computing, 1978, pp. 171-178.

[5] J. Nesetril and V. Rodl, The structure of critical Ramsey graphs, vol. 32, Acta Mathematica Hungaria, 1978, pp. 295-300.

[6] T. Vetrik, L. Yulianti, E.T. Baskoro, On Ramsey $\left(K_{1,2}, C_{4}\right)$-minimal graphs, vol. 30, Discussiones Mathematicae Graph Theory, 2010, pp. 637-649.

[7] F.F. Hadiputra, D.R. Silaban, Infinite Family of Ramsey $\left(K_{1,2}, C_{4}\right)$-minimal Graphs, vol. 1722, Journal of Physics: Conference Series, 2021. DOI: 10.1088/1742-6596/1722/1/012049.

[8] M. Nabila, E.T. Baskoro, On Ramsey $\left(C_{n}, H\right)$ minimal graphs, vol.1722, Journal of Physics: Conference Series, 2021, pp. DOI: 10.1088/17426596/1722/1/012049. 